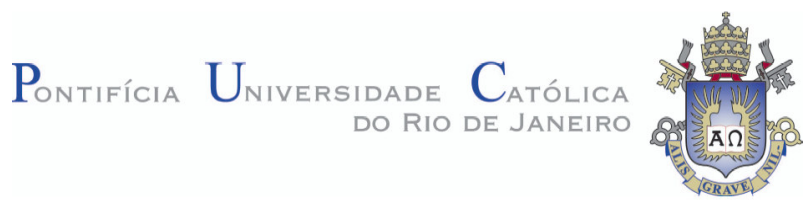

Vinicius Pimenta de Avellar

\title{
Modelagem do Regime Transitório de Turbinas a Gás Industriais para a Geração de Energia Elétrica
}

Dissertação apresentada ao Programa de Pósgraduação em Engenharia Mecânica da PUC-Rio como requisito parcial para obtenção do título de Mestre em Engenharia Mecânica.

Orientador: Prof. Sergio Leal Braga Co-orientador: Prof. Sandro Barros Ferreira

Rio de Janeiro

Fevereiro de 2010 


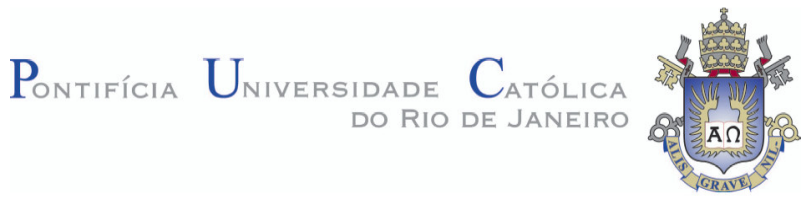

Vinicius Pimenta de Avellar

\title{
Modelagem do Regime Transitório de Turbinas a Gás Industriais para a Geração de Energia Elétrica
}

\begin{abstract}
Dissertação apresentada como requisito parcial para obtenção do grau de Mestre pelo Programa de Pósgraduação em Engenharia Mecânica do Centro Técnico Científico da PUC-Rio. Aprovado pela Comissão Examinadora abaixo assinada.
\end{abstract}

Prof. Sergio Leal Braga

Orientador

Departamento de Engenharia Mecânica - PUC-Rio

Prof. Sandro Barros Ferreira

Co-orientador

GT2 Energia

Prof. Luís Fernando Figueira da Silva

Departamento de Engenharia Mecânica - PUC-Rio

Prof. Marco Aurélio da Cunha Alves

Instituto de Aeronáutica e Espaço

Prof. José Eugenio Leal

Coordenador Setorial do Centro Técnico Científico - PUC-Rio

Rio de Janeiro, 26 de fevereiro de 2010 
Todos os direitos reservados. É proibida a reprodução total ou parcial do trabalho sem autorização da universidade, do autor e do orientador.

\section{Vinicius Pimenta de Avellar}

Graduou-se em Engenharia Elétrica com ênfase em Sistema de Energia Elétrica e Sistema de Apoio a Decisão na Pontifícia Universidade Católica do Rio de Janeiro em 2005.

Ficha catalográfica

Avellar, Vinicius Pimenta de

Modelagem do regime transitório de turbinas a gás industriais para a geração de energia elétrica / Vinicius Pimenta de Avellar; orientador: Sergio Leal Braga; co-orientador: Sandro Barros Ferreira. - 2010

95 f.: II. (color) ; $30 \mathrm{~cm}$

Dissertação (Mestrado em Engenharia Mecânica) - Pontifícia Universidade Católica do Rio de Janeiro, Rio de Janeiro, 2010.

Inclui bibliografia.

1. Engenharia mecânica - Teses. 2. Turbinas a gás. 3. Sistemas de controle. 4. Modelagem do regime transitório. I. Braga, Sergio Leal. II. Ferreira, Sandro Barros. III. Pontifícia Universidade Católica do Rio de Janeiro Departamento de Engenharia Mecânica. IV. Título.

CDD: 621 
Às pessoas que não mediram esforços para me educar: meus pais. 


\section{Agradecimentos}

Ao Dr. Sandro Barros Ferreira pela confiança, oportunidade, pelo constate apoio nos momentos de dificuldade e pela orientação ao longo da dissertação deste trabalho.

Ao Professor Marco Aurélio da Cunha Alves pelo ensinamento passado durante o nosso convívio, e por compartilhar o seu programa de simulação.

Ao Professor Sergio Leal Braga no auxilio para ingressar no curso de pósgraduação da PUC-Rio.

Ao Professor Luis Fernando Figueira da Silva por ajudar na redação deste trabalho.

Ao Clóvis Henrique Meirelles Fonseca pela ajuda na obtenção dos dados utilizados na validação do modelo desenvolvido.

A minha família pelo apoio incondicional durante a minha vida. 


\section{Resumo}

Avellar, Vinicius Pimenta de; Braga, Sergio Leal. Modelagem do Regime Transitório de Turbinas a Gás Industriais para a Geração de Energia Elétrica. Rio de Janeiro, 2010. 95p. Dissertação de Mestrado Departamento de Engenharia Mecânica, Pontifícia Universidade Católica do Rio de Janeiro.

As turbinas a gás são equipamentos de vital importância para o setor industrial, fornecendo trabalho e calor para diversos setores, do transporte aos sistemas de cogeração. A crescente necessidade de geração de energia elétrica confiável tem incentivado o projeto de turbinas a gás industriais, inclusive no Brasil, que operam com vários combustíveis como o diesel, gás natural, álcool e de combustíveis de baixo poder calorífico. Para melhor monitorar e controlar estes motores, uma análise completa da previsão de funcionamento em regime transitório é necessária. Durante o regime transitório das turbinas a gás industriais (heavy-duty), o sistema de controle deve manter os limites de certos parâmetros, tais como a temperatura na entrada da turbina e a velocidade de rotação do eixo, no seu valor nominal. Além disso, o tempo de resposta necessário para o sistema de controle atuar deve ser o mais breve possível para garantir uma operação de qualidade, segura e confiável. A temperatura de entrada da turbina, que é um parâmetro muito importante no desempenho de uma turbina a gás, é limitada pela resistência mecânica do material das pás da turbina. A velocidade de rotação do eixo deve permanecer constante, devido à ligação ao sistema elétrico, que não pode suportar altas flutuações de frequiência. Este trabalho tem como motivação o incremento da capacidade de simulação de um modelo computacional existente, incorporando, para este fim, rotinas de sistemas de controle. Como resultado, o novo modelo é capaz de simular qualquer condição de funcionamento de turbinas a gás industriais, em regime permanente e transitório controlado. Os resultados obtidos pelo programa computacional se mostraram fiéis ao comportamento real da máquina. Além disso, mostraram a flexibilidade do modelo ao lidar com diferentes condições de operação.Um programa computacional capaz de simular o desempenho transitório controlado de turbinas a gás é de extrema relevância para 
o desenvolvimento de softwares que auxiliam os operadores destes equipamentos.

Dentre estes, estão os sistemas de monitoramento e diagnóstico dos equipamentos em questão.

\section{Palavras-chave}

Turbina a gás; sistema de controle; modelagem do regime transitório. 


\section{Abstract}

Avellar, Vinicius Pimenta de; Braga, Sergio Leal (Advisor). Transient Modelling of Industrial Gas Turbine for Power Generation. Rio de Janeiro, 2010. 95p. MSc. Dissertation - Departamento de Engenharia Mecânica, Pontifícia Universidade Católica do Rio de Janeiro.

Gas turbine engines are a vital part of today's industry, providing both work and heat for several industry sectors, from transportation to cogeneration systems. The growing need for reliable electricity has encouraged the design of stationary gas turbines, including in Brazil, which operates on multiple fuels such as diesel, natural gas and low calorific fuels. To better monitor and control these engines, a complete analysis for prediction of transient operation is required. During transient operation of heavy duty gas turbines, the control system must keep the limits of certain parameters, such as turbine inlet temperature (TIT) and the rotational shaft speed within their design range. Moreover, the time required for the control system to react should be as short as possible to guarantee a safe and reliable operation. The turbine inlet temperature, which is a very important parameter in the performance of a gas turbine, is limited by the turbine blades material mechanical resistance. Furthermore, the rotational speed should remain constant due to the electric grid connection, which cannot withstand high frequency fluctuations. This work is motivated by the need to increase the ability of a computer model to simulate the performance of industrial gas turbines, incorporating, for this purpose, control system routines. As a result, the new model will be able to simulate any operating condition of industrial gas turbines, in both steady state and transient. The results obtained by the computer program proved to be faithful to the actual behavior of the engine. Furthermore, they showed the flexibility of the model to deal with different operating conditions. A computer program capable of simulating the transient performance of gas turbines is very important for the development softwares to help operators of such equipment. In addition, it could be used in on-line intelligent diagnostic program.

\section{Keywords}

Gas turbine; control system; transient regime simulation. 


\section{Sumário}

1 Introdução

2 Revisão Bibliográfica

3 Regime Permanente de Turbinas a Gás

3.1. Desempenho de Turbinas a Gás em Ponto de Projeto

3.1.1. Introdução

3.1.2. Resultados da Simulação da Turbina a Gás Operando no Ponto de Projeto

3.2. Desempenho de Turbinas a Gás Operando Fora do

Ponto de Projeto - Plena Carga

3.2.1. Resultados da Simulação em Plena Carga

3.3. Desempenho da Turbina a Gás Operando Fora do

Ponto de Projeto - Carga Parcial

3.3.1. Resultados

4 Regime Transitório de Turbinas a Gás

4.1. Introdução

4.2. Análise da Dinâmica dos Gases

4.2.1. Equação de Conservação de Massa

4.2.2. Equação da Conservação de Energia

4.2.3. Equação da Quantidade de Movimento

4.2.4. Modificação das Equações de Conservação

4.3. Transitório de Eixo

5 Controle de Turbinas a Gás

5.1. Introdução

59

5.2. Controle de Processos

62

5.3. Controlador 
5.5. Resultados e Discussões

$\begin{array}{ll}6 \text { Conclusões } & 80\end{array}$

7 Trabalhos Futuros 83

8 Referências bibliográficas $\quad 84$

9 Anexo $\quad 86$ 


\section{Lista de figuras}

Figura 1 - Diagrama de blocos do controle da turbina a gás descrito em Rowen [2].

Figura 2 - Diagrama de blocos do novo controle da turbina a gás descrito em Rowen [3].

Figura 3 - Comparação dos resultados da turbina a gás usando o programa de simulação descrito por Agresti et al. [5], linhas tracejadas, como os obtidos por Rowen [2], linhas contínuas. 25 Figura 4 - Comparação entre o modelo simulado e os dados de operação de uma turbina a gás GE 7F em regime transitório: (a) potência gerada; (b) desvio da rotação nominal; (c) vazão mássica de combustível; (d) temperatura de entrada do rotor da turbina; (e) temperatura de exaustão da turbina a gás;

(f) ângulo das VIGVs Kim et al. [6].

Figura 5 - Diagrama de blocos do controle da turbina a gás descrito em Kim et al. [6].

Figura 6 - Esquema simplificado de uma turbina a gás de um eixo Camporeale et al. [8].

Figura 7 - Resultados normalizados para a turbina a gás de um eixo: (a) carga aplicada; (b) variáveis controladas;

(c) variáveis de controle; (d) temperatura e pressão na descarga do compressor $\left(T_{2}\right.$ e $\left.p_{2}\right)$ e saída do combustor $\left(T_{3}\right.$ e $\left.p_{3}\right)$

Camporeale et al. [8].

Figura 8 - Resultados da simulação para a turbina a gás de eixo duplo: (a) variação da carga gerada; (b) velocidade rotacional do gerador de gás e da turbina de potência;

(c) sinal de controle VCE e vazão mássica de combustível;

(d) mapa do compressor durante o transiente; (e) valores de temperatura em alguns pontos do motor; $(f)$ valores de pressão em alguns pontos do motor Camporeale et al. [8].

Figura 9 - Comparação da resposta dos modelos propostos 
por Rowen [2] e pela IEEE Yee et al. [10].

Figura 10 - Diagrama de blocos representando a modelagem

do ciclo combinado por Bagnasco et al.[11].

Figura 11 - Resultado do modelo proposto por Bagnasco et al. [11]. 36

Figura 12 - Comparação entre a vazão mássica de exaustão simulada pelo modelo e os dados fornecidos pela usina.

Figura 13 - Comparação entre o heat consumption calculado pelo modelo e os dados fornecidos pela usina.

Figura 14 - Comparação entre o heat rate calculado pelo modelo e os dados fornecidos pela usina.

Figura 15 - Comparação entre a potência gerada calculada pelo modelo e os dados fornecidos pela usina.

Figura 16 - Efeito das VIGVs na vazão mássica de exaustão em função da potência gerada.

Figura 17 - Efeito das VIGVs na vazão mássica de exaustão em função da potência gerada.

Figura 18 - Mapa de característica do compressor deslocado devido à variação das VIGVs.

Figura 19 - Temperatura de exaustão da turbina a gás comparação entre os resultados do modelo computacional e os dados fornecidos pela usina para uma temperatura ambiente de $15^{\circ} \mathrm{C}$.

Figura 20 - Vazão mássica de exaustão da turbina a gás comparação entre os resultados do modelo computacional e os dados fornecidos pela usina para uma temperatura ambiente de $15^{\circ} \mathrm{C}$.

Figura 21 - Diagrama esquemático para o desenvolvimento da equação da conservação de massa para um volume de controle. Figura 22 - Diagrama esquemático para o desenvolvimento da equação de conservação de energia para um volume de controle. $\quad 54$ Figura 23 - Variáveis manipuladas da turbina a gás industrial.

Figura 24 - Diagrama esquemático do sistema de controle de malha aberta. 
Figura 25 - Diagrama esquemático do sistema de controle de malha fechada.

Figura 26 - Diagrama esquemático do sistema de controle da velocidade de rotação do eixo a partir da manipulação da injeção de combustível.

Figura 27 - Respostas da velocidade de rotação do eixo a variações de carga com sistema de controle.

Figura 28 - Variação da velocidade de rotação do eixo para variações de carga sem sistema de controle.

Figura 29 - Diagrama de blocos do sistema de controle implementado.

Figura 30 - Transitório de potência - dados de uma turbina a gás em operação.

Figura 31 - Comparação entre os resultados de ângulo das VIGVs simulados e os fornecidos pela usina.

Figura 32 - Comparação entre os resultados de ângulo das VIGVs simulados e os fornecidos pela usina, durante o período transitório.

Figura 33 - Erro percentual do ângulo das VIGVs.

Figura 34 - Comparação entre os resultados de vazão de combustível simulados e os dados fornecidos pela usina.

Figura 35 - Comparação entre os resultados do modelo e os dados de operação da turbina a gás no período de discrepância, referente a vazão mássica de combustível.

Figura 36 - Oscilações capturadas pelo modelo computacional, referente a vazão mássica de combustível.

Figura 37 - Erro percentual da vazão de combustível.

Figura 38 - Comparação entre os resultados de pressão de descarga do compressor simulados e os dados fornecidos pela usina. 74 Figura 39 - Oscilações capturadas pelo modelo computacional, referente à pressão de descarga do compressor. 75 Figura 40 - Erro percentual da pressão de descarga do compressor. 75 Figura 41 - Comparação entre os resultados de temperatura de exaustão simulados e os dados fornecidos pela usina. 
Figura 42 - Erro percentual da temperatura de exaustão da $t$ urbina a gás.

Figura 43 - Temperatura de exaustão da turbina a gás.

Figura 44 - Comparação entre os resultados de velocidade de rotação do eixo simulados e os dados fornecidos pela usina.

Figura 45 - Visualização das oscilações capturadas pelo modelo computacional, referente à velocidade de rotação do eixo.

Figura 46 - Erro percentual da velocidade de rotação do eixo da turbina a gás. 


\section{Lista de tabelas}

Tabela 1 - Dados de desempenho da turbina a gás opernado em condições de ponto de projeto.

Tabela 2 - Dados de entrada do programa computacional.

Tabela 3 - Resultados da simulação do ponto de projeto da

turbina a gás analisada.

Tabela 4 - Desvios percentuais dos resultados do modelo computacional comparados com os dados de catálogo da turbina a gás em estudo, operando fora do ponto de projeto em plena carga.

Tabela 5 - Desvios percentuais dos resultados do modelo computacional comparados com os dados de catálogo da turbina a gás em estudo, operando fora do ponto de projeto em plena carga.

Tabela 6 - Ganho dos controladores utilizados no modelo 


\title{
Nomenclatura
}

\author{
A Área \\ C Velocidade do fluido \\ $C_{P} \quad$ Calor específico a pressão constante \\ $C_{P G} \quad$ Calor específico dos gases produtos da combustão \\ $C_{V} \quad$ Calor específico a volume constante \\ E Energia total \\ $f \quad$ Relação combustível/ar \\ $g \quad$ Aceleração da gravidade \\ h Entalpia \\ I Momento de inércia \\ $K_{p} \quad$ Ganho proporcional \\ $L \quad$ Quantidade de movimento angular \\ $\dot{m} \quad$ Vazão mássica \\ M Número de Mach \\ $N \quad$ Velocidade de rotação do eixo \\ p Pressão \\ PCI Poder calorífico inferior do combustível \\ $\dot{Q} \quad$ Taxa de transferência de calor \\ $r \quad$ Raio médio do rotor \\ $R \quad$ Constante universal dos gases \\ $t \quad$ Tempo \\ $T \quad$ Temperatura \\ $T_{I} \quad$ Tempo integrativo \\ $T_{D} \quad$ Tempo derivativo \\ $U \quad$ Velocidade tangencial à palheta do rotor \\ V Volume \\ $\dot{W} \quad$ Taxa de transferência de trabalho - potência \\ w Trabalho específico / Velocidade angular
}


Altura

\section{Letras gregas}

$\alpha \quad$ Aceleração angular

$\gamma \quad$ Razão entre o calor específico a pressão constante e o calor específico a volume constante

$\begin{array}{ll}\eta & \text { Eficiência } \\ \rho & \text { Densidade do gás } \\ \tau & \text { Torque } \\ \text { Índices } & \\ b & \text { Combustão } \\ c & \text { Compressor } \\ f & \text { Combustível } \\ t & \text { Turbina } \\ 0 & \text { Propriedade de estagnação } \\ s & \text { Eixo }\end{array}$

